\title{
Preparation of monoclonal antibody bank against whole water-soluble proteins from rapid-growing bamboo shoots
}

\author{
Yu-Jen $W u^{1,2}$, Han-Min Chen ${ }^{3 *}$, Tai-Tse Wu ${ }^{1 *}$, Jiann-Shing $W^{1^{*}}$, Rea-Min Chu ${ }^{4}$ \\ and Rong-Huay Juang ${ }^{1}$ \\ ${ }^{1}$ Department of Biochemical Science and Technology, Institute of Microbiology and Biochemistry, \\ National Taiwan University, Taipei, Taiwan \\ 2 Department of Beauty Science, Meiho Institute of Technology, Pingtung, Taiwan \\ ${ }^{3}$ Department of Life Science, Catholic Fu-Jen University, Taipei, Taiwan \\ ${ }^{4}$ Department of Veterinary Medicine, National Taiwan University, Taipei, Taiwan
}

\begin{abstract}
An antibody bank against the whole proteins in a proteome is a useful tool for biological research. Using the standard cell fusion method, and a modified screening protocol, we produced an $\mathrm{mAb}$ bank against the total water-soluble proteins extracted from the rapid-growing green bamboo shoots. An improved two-stage strategy was employed to enrich those poor immunogenic or lower expressed proteins. Totally, we obtained a bank of $192 \mathrm{mAb}$ which were identified as distinctive to each other by 2-DE and immunostaining.
\end{abstract}

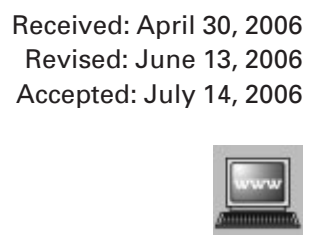

\section{Keywords:}

2-DE immunostaining / Bambusa oldhamii / Green bamboo shoots / Hybridoma / Monoclonal antibody bank

When proteomic research brings in powerful analytical techniques such as 2-DE for separation and MS for protein identification, a specific probe to the target spots on the 2-DE gel is urgently required $[1,2]$. Although a computer software could help to some extent, it is a tedious work to compare the protein spots on the 2-DE gels, especially when the proteomes are too complex to resolve the spots unambiguously. Antibody is a specific probe to its antigen, and could be produced by immunizing the antigen against an animal host of different species. Each antibody is secreted by its specific Bcell line which could be immortalized by fusing with the myeloma cells; and the resulting hybridoma cell line produces a single $\mathrm{mAb}$ which recognizes only its specific anti-

Correspondence: Professor Rong-Huay Juang, Department of Biochemical Science and Technology, Institute of Microbiology and Biochemistry, National Taiwan University, Taipei 106, Taiwan E-mail: juang@ntu.edu.tw

Fax: +86-62-3366-4081

Abbreviation: IgG, immunoglobulin G genic determinant [3]. Theoretically the host will produce antibody against every invading protein which contains a peptide with a different amino acid sequence. Therefore, if the total proteins of the foreign tissue are used for immunization, it is possible to produce a bank of $\mathrm{mAb}$ against most of the proteins in that proteome [4]. However, some poor antigens, either with weak immunogenicity or low abundance, might not elicit immune response effectively. In such circumstances, affinity chromatographic removal of the proteins, which induces antibodies readily in the first-stage of immunization, might be a good strategy to enrich those poor antigens [5]. Second-stage immunization of the purified proteins to another mouse should increase the opportunity to activate the corresponding B-cell for antibody production. If necessary, more cycles of adsorption and immunization could be performed. Putting these strategies together, we prepared an $\mathrm{mAb}$ bank against the total proteins from the shoots of green bamboo (Bambusa oldhamii) using a multiple stage protocol (Fig. 1A). The final mAb bank will be useful in

* These authors contributed equally to this work. 
A

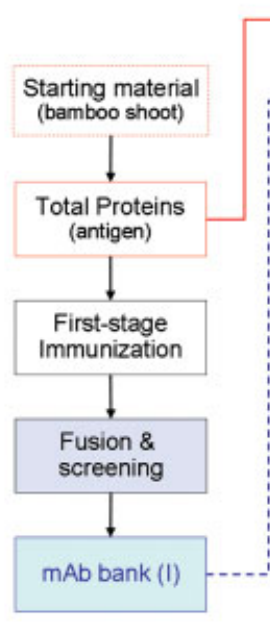

First stage

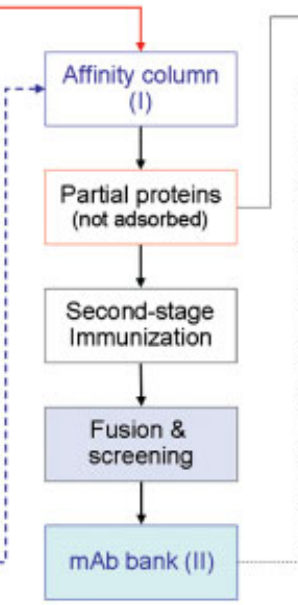

Second stage

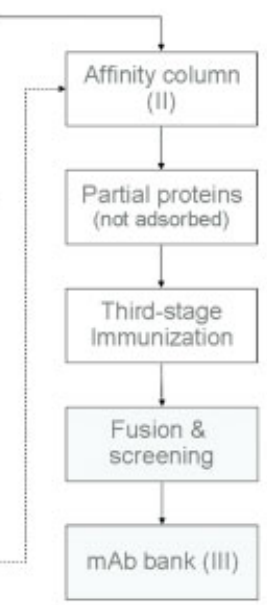

(Third stage)
B

Do

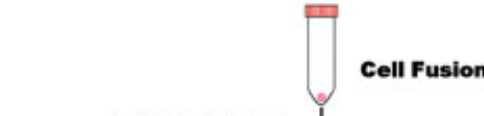

D14

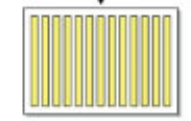
Master plales (3 plates)

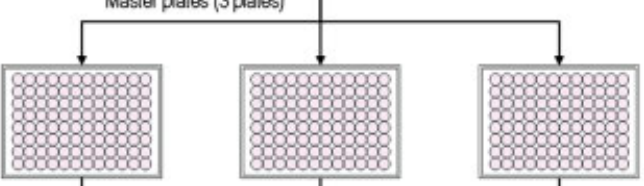

D21

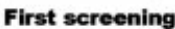

1.DE immunostaining

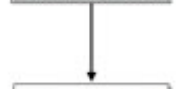

28

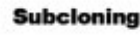

(1 plate per clone)
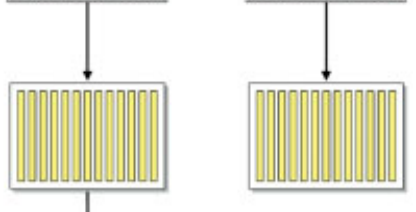

-25 culture

Positive clone

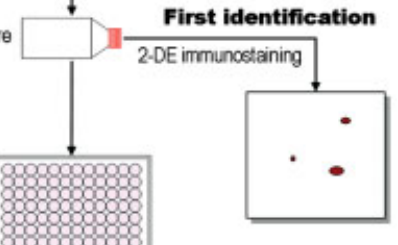

D42
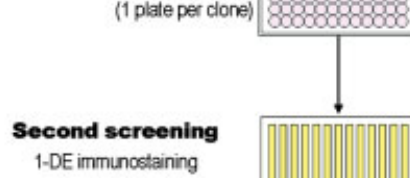

D49
1-DE immunostaining
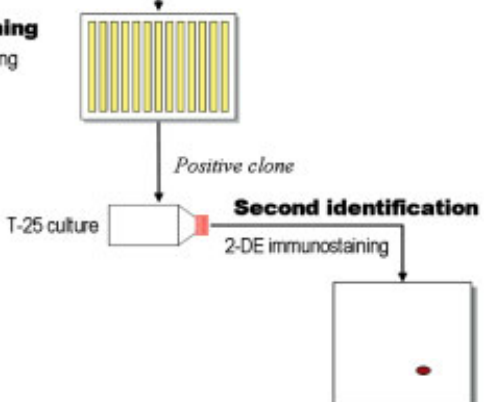

Figure 1. Flow charts for the stage-wise production of mAb bank. (A) Each stage consists of the immunization, fusion, and screening steps for an antigen with complex proteins. The proteins which induced antibodies successfully in the first stage were removed by affinity adsorbent immobilized with all the first-stage mAb. The remaining proteins were then used as the antigen for the second stage. We have carried out only two stages in this study. (B) The detailed procedure for screening mAb produced clones in each stage. An approximate time schedule was shown on the left, which was started from the day of fusion (D0). For the screening steps, the 1-D SDS-PAGE (1-DE) and immunostaining was used instead of ELISA in the conventional hybridoma screening. Since the antigen was a mixture of proteins in which the minor proteins might be masked by the dominant proteins when coated in the ELISA wells. The binding specificity for each individual mAb was examined by 2-DE immunostaining in the identification steps. Some clones might show identical staining patterns on their 2-DE blots, only one was kept in the final bank.

investigating the expression of proteins during the rapid growth of bamboo shoots, which will be published elsewhere.

The total water-soluble proteins of the bamboo shoot $(60 \mathrm{~cm}$ height above the ground) were extracted and analyzed by 2 -DE (Fig. 2A). The extraction of total proteins followed the method described by Gegenheimer [6]. An aliquot of the total proteins $(50 \mu \mathrm{g})$ was used to immunize a Balb/c mouse every $2 \mathrm{wk}$ for 3 months; mouse sera were collected during the immunization, and examined by 2-DE immunostaining. For the first-stage $\mathrm{mAb}$ bank preparation, two mice (mouse $\mathrm{A}$ and mouse B) were immunized following the same immu- nization protocol. The titer started to increase after $6 \mathrm{wk}$, and reached the maximum at 12 th week (Supplementary Fig. 1). The mice were sacrificed after the final immunization, their spleen cells were collected and fused with myeloma NSO/1 (mouse A) and Sp2/0 (mouse B), respectively. Cell fusion was carried out by a standard procedure which was described in our previous report [7]. After the fusion, each cell mixture was distributed into three 96-well plates which were cultured immediately under HAT (hypoxanthine-aminopterin-thymidine) selection (D0, Fig. 1B). On D14, supernatant from each well was sampled and analyzed by 1-DE (12.5\% SDS-PAGE) and immunostaining (first screening). Positive clones were 


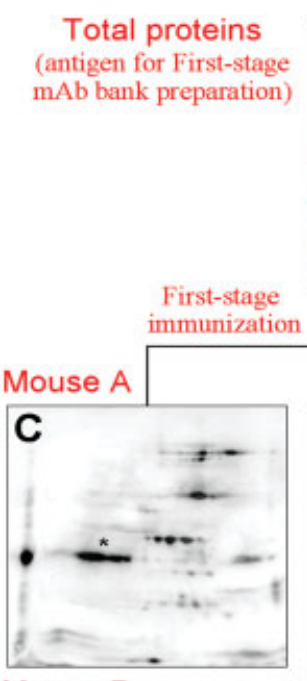

Mouse B

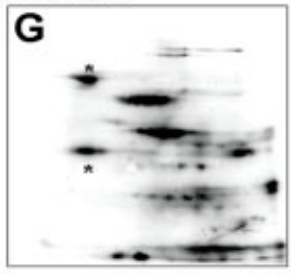

Antiserum

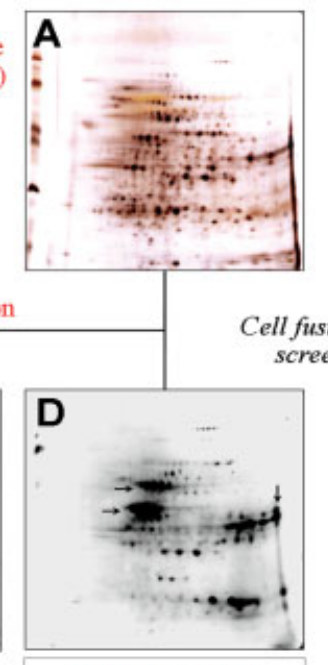

First-stage bank (mixture of $160 \mathrm{mAb}$ )

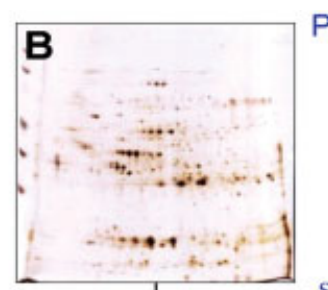
Second-stage
immunization
Proteins after absorption

(antigen for Second-stage

mAb bank preparation)

\section{Mouse C}

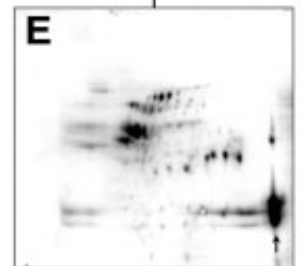

Second-stage bank (mixture of $32 \mathrm{mAb}$ )

F

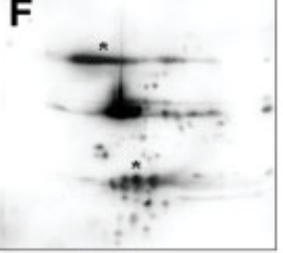

Antiserum

Total mAb bank

(mixture of 192 clones)

I 2-DE-immunostaining blots of $192 \mathrm{mAb}$

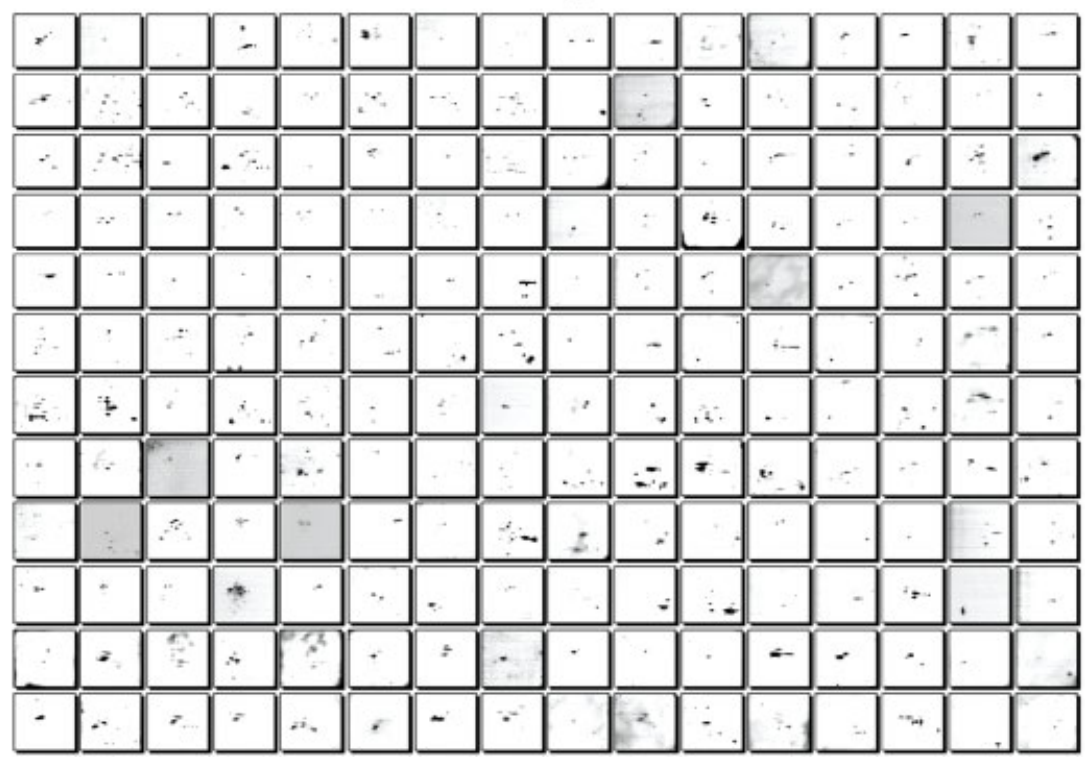

Figure 2. The immune response against the complex protein antigens from the rapid-growing bamboo shoots was monitored by 2-DE and immunostaining. The silver-staining patterns of 2-DE gels for the total proteins ( $A$, used for the first-stage antigen) and the remaining proteins after the affinity adsorption ( $B$, for the second-stage antigen) were compared. Mouse antisera were collected after their immunization, and used to stain the first-stage ( $C$ and $G$ ) and the second-stage antigens ( $F$ ), respectively. On the other hand, blots stained by $m A b$ mixtures from the first-stage bank (D) and the second-stage bank (E) showed distinctive patterns to each other. Finally, the total $192 \mathrm{mAb}$ from these two banks were mixed to reveal the whole binding pattern $(H)$, which looked similar to the total protein pattern $(A)$ but not identical. Some differences between these blots were marked with asterisks and arrows which were described in the text. (I) Each individual mAb showed a unique staining pattern on the 2-DE blot. Larger images of the 192 blots are displayed in Supplementary Fig. 2. 
picked and expanded to T-25 culture, and the medium was used to reveal the binding pattern of the mAb by $2-\mathrm{DE}$ immunostaining (first identification). Candidate clones were selected based on the first identification, and were further subcloned by limiting the dilution (one 96-well plate per candidate). Positive clones were screened again by 1-D immunostaining (second screening), and then expanded to T-25 for the final confirmation by 2-DE immunostaining (second identification). Clones targeting the same antigen were identified and rejected by examining their 2-DE immunostaining patterns during the identification steps.

The supernatant of the final hybridoma cell culture was collected for antibody purification. The immunoglobulin (IgG) was fractionated by ammonium sulfate precipitation at $40 \%$ saturation. The precipitate was dissolved in $50 \mathrm{mM}$ $\mathrm{NaHCO}_{3}$ buffer ( $\mathrm{pH}$ 8.3), and then dialyzed at $4^{\circ} \mathrm{C}$ overnight. The precipitate formed during dialysis was removed by centrifugation before applying to the DEAE-Sephacel column (bed volume $10 \mathrm{~mL}$ equilibrated in $\mathrm{NaHCO}_{3}$ buffer). The column was washed with several volumes of $150 \mathrm{mM} \mathrm{NaCl}$ in $\mathrm{NaHCO}_{3}$ buffer, and then the IgG fraction was eluted out with $200 \mathrm{mM} \mathrm{NaCl}$ in the same buffer. The IgG was then immobilized on CNBr-Sepharose (Pharmacia) according to a method from the supplier. Totally, we prepared 160 individual affinity adsorbents for every $\mathrm{mAb}$ in the first-stage bank. Half milliliter of the affinity adsorbent from each $\mathrm{mAb}$ was pooled together and packed into a column (id $2.5 \mathrm{~cm} \times 16 \mathrm{~cm})$. The total soluble proteins $(\mathrm{ca} .20 \mathrm{~g})$ extracted from the bamboo shoots were applied to this column, the eluent was collected and applied immediately back to the column. This application process was repeated eight times to adsorb the first-stage antigens in the total proteins. The final proteins that flowed through the affinity column were collected and concentrated by Centriplus (Amicon), which were then used in immunizing another mouse (mouse C) for the preparation of the second-stage $\mathrm{mAb}$ bank following the same protocol as the first-stage (Fig. 1A).
The results of the fusion and selection are summarized in Table 1. After the first-stage cell fusion, about one-third of the microtiter wells showed positive on 1-D blots (first screening). While each positive well might contain more than one cell line, the positive numbers expanded to 1.5- or 2-fold after subcloning, and totally 400 clones were positive (second screening). However, some clones showed identical 2-DE patterns (second identification), and only one of them was kept in the final mAb bank. Therefore, the final number of the first-stage bank shrunk to 160 , in which mouse A produced 78 unique clones, whereas mouse B contributed 82 of them. For the second stage, only 40 wells (14\%) appeared positive after the first screening. It is due to the fact that the protein repertoire used as the antigen in this stage was simpler and less immunogenic. After the subcloning and second identification, 32 unique clones were selected and proved to be distinguished from the cell lines of the first stage. Totally, we obtained $192 \mathrm{mAb}$ showing distinctive 2-DE staining patterns (Fig. 2I, or larger images in Supplementary Fig. 2). It is noticeable that most $\mathrm{mAb}$ in this bank stained more than one spot on its 2-DE blot. Some unique clones (e.g., AE6B12 in Supplementary Fig. 2A) showed very complex patterns which appeared to be stained with a polyclonal antiserum. We have checked these clones further by another subcloning step, and confirmed that they were monoclonal. For an approximate estimation, about 70 lines (36\%) showed simple staining patterns (less than three spots), and 40 lines (21\%) obtained complex patterns like AE6B12. The total number of spots recognized by the first-stage $\mathrm{mAb}$ bank reached 1140 by summing up all the 160 blots, and 220 for the second-stage. On an average, every $\mathrm{mAb}$ can stain up to seven spots on the 2-DE. This observation might illustrate the complexity of the protein modification after the translation. Another explanation is that several independent proteins might share a common antigenic determinant which could be recognized by the same mAb.

Table 1. Summary of the two stages for mAb bank production

\begin{tabular}{|c|c|c|c|c|}
\hline \multirow{2}{*}{$\begin{array}{l}\text { Spleen cells fused with number } \\
\text { of clones }\end{array}$} & \multicolumn{2}{|c|}{ NS0/1 (mouse A) ${ }^{a)}$} & \multicolumn{2}{|c|}{ Sp2/0 (mouse B) $)^{a}$} \\
\hline & Screened & Positive & Screened & Positive \\
\hline \multicolumn{5}{|l|}{ (A) First-stage bank } \\
\hline First screening & 150 & 100 & 180 & 120 \\
\hline Second screening (after subcloning) & 320 & 150 & 500 & 250 \\
\hline Final monoclonal clones & & 78 & & 82 \\
\hline \multicolumn{5}{|l|}{ (B) Second-stage bank for mouse $C^{\text {a) }}$} \\
\hline First screening & & & 100 & 40 \\
\hline Second screening (after subcloning) & & & 400 & 120 \\
\hline Final monoclonal clones & & & & 32 \\
\hline
\end{tabular}

a) After cell fusion, the cell mixture was distributed into three 96-well microtiter plates. 
To check for the total coverage of protein spots recognized by $\mathrm{mAb}$ in the bank, the mixture of $160 \mathrm{mAb}$ produced in the first stage was used for staining the total proteins on the 2-DE blot. The resulting pattern (Fig. 2D) revealed only a part of the total protein spots of the silver-stained gel (Fig. 2A), since weak immunogenic or low abundant proteins could not induce $\mathrm{Ab}$ efficiently. Nevertheless, some highly immunogenic proteins produced $\mathrm{mAb}$ with enhanced titer in this stage (indicated with horizontal arrows in Fig. 2D). The affinity adsorbent immobilized with these 160 $\mathrm{mAb}$ did remove a great part of the immunogens, as compared to the 2-DE patterns of the second-stage antigens (Fig. 2B) with those of the first-stage (Fig. 2A). Accordingly, the immunostaining pattern using the mixture of the second-stage $\mathrm{mAb}$ (Fig. 2E) was significantly different from the first-stage bank (Fig. 2D). When we further compared the pattern stained by a mixture of all $192 \mathrm{mAb}$ (Fig. 2H) with the total proteins (Fig. 2A), there were still some proteins which could not produce antibody throughout these two stages. However, immunostaining patterns by mouse antisera collected during the immunization (Figs. 2C, G and F) did stain additional spots (examples are marked with asterisks). We might lose these clones during the screening and subcloning procedure. On the other hand, some spots were stained strongly only with $\mathrm{mAb}$, but not with antisera (examples indicated with vertical arrows in Figs. D and E).

In the first stage, we immunized two Balb/c mice with the same antigens for 3 months; the immune responses were rather different as revealed from their 2-DE patterns by antisera (Figs. C and G). Serum from mouse B showed higher titer and a more complex staining pattern than that from mouse A. However, these two mice produced nearly equal number of $\mathrm{mAb}$ in the first-stage banks (Table A). Based on this observation, we might increase the number of mouse in the second-stage immunization to produce a broader spectrum of $\mathrm{mAb}$ against weak antigens. In this study, only the IgG-secreting cell lines were selected for the mAb bank, since we used the antimouse IgG second antibody in all screening procedures. The IgG subclass was intentionally selected, because it was monomeric, and was induced in the later period of immunization, which might generate a higher affinity against the antigens through the somatic mutation and affinity maturation.

Recent reviews [1, 2] have suggested the urgent need of specific probes for the identification of unknown proteins in a proteome. Instead of the hybridoma technique, phage antibody [8] is generally regarded as the tool of choice for producing an antibody bank. Liu et al. [9] have demonstrated a proteome-wide antibody production by phage display, in which dozens of unique mAb were selected. The major obstacle for the hybridoma technique is its redundant and time-consuming work. Besides, some minor proteins have difficulty in inducing antibody since they are poor immunogenic or insufficiently expressed. Nevertheless, immune response in the vertebrate is the most effective way for producing specific binding molecules against a defined target. To the best of our knowledge, at least one group has shown the possibility of producing about $50 \mathrm{mAb}$, by standard immunization and cell fusion technique against the mitochondrial proteins from human liver [4]. And the antigen enrichment by adsorbing several major proteins in the plasma has been shown by another study [5]. We stepped further by putting these strategies together and using modified screening protocols, produced nearly 200 individual $\mathrm{mAb}$ against the whole proteins from bamboo shoots by a two-stage protocol, which could enrich the poor antigen effectively. In total, three mice were sacrificed in the whole experiment, which was completed in 18 months by two fulltime research staffs. The $\mathrm{mAb}$ repertoire should increase if additional stages were performed. The next step was to identify the specific antigen for each mAb that could be accomplished by immunoprecipitation and MS as described by other groups $[4,5]$. The application with hundreds of unique $\mathrm{mAb}$ in analyzing important proteins on cancer tissues $[10,11]$, or preparing the antibody array [12] for the proteomic research, is thus practical and promising.

This work was supported by the Council of Agriculture, R.O.C. (Taiwan).

\section{References}

[1] Konthur, Z., Hust, M., Dubel, S., Gene 2005, 364, 19-29.

[2] Hanash, S., Mol. Cell. Proteomics 2004, 3, 298-301.

[3] Kohler, G., Milstein, C., Nature 1975, 256, 495-497.

[4] Gao, J., Gao, Y., Ju, Y., Yang, J. et al., Proteomics 2006, 6, 427-437.

[5] Ning, Y., Wang, Y., Li, Y., Hong, Y. et al., Proteomics 2006, 6, 438-448.

[6] Gegenheimer, P., Methods Enzymol. 1990, 182, 174-193.

[7] Chen, H. M., Chang, S. C., Wu, C. C., Cuo, T. S. et al., Physiol. Plant. 2002, 114, 506-515.

[8] McCafferty, J., Griffiths, A. D., Winter, G., Chiswell, D. J., Nature 1990, 348, 552-554.

[9] Liu, B., Huang, L., Sihlbom, C., Burlingame, A. et al., J. Mol. Biol. 2002, 315, 1063-1073.

[10] Uhlen, M., Ponten, F., Mol. Cell. Proteomics 2005, 4, 384-393.

[11] Uhlen, M., Bjorling, E., Agaton, C., Szigyarto, C. A. et al., Mol. Cell. Proteomics 2005, 4, 1920-1932.

[12] Haab, B. B., Mol. Cell. Proteomics 2005, 4, 377-383. 